Mieczysław C. Paczkowski OFM

Uniwersytet Mikołaja Kopernika w Toruniu celestyn@umk.pl

ORCID: 0000-0002-4045-2314

DOI: http://dx.doi.org/10.12775/BPTh.2019.003
Biblica

et

Patristica

Thoruniensia

12 (2019) 1: 43-69

ISSN (print) 1689-5150

ISSN (online) 2450-7059

\title{
Esegesi patristica di Gv 4,21-24 \\ e il vero culto a Gerusalemme (II parte)
}

\section{The patristic exegesis of Joh 4:21-24 and the true worship in Jerusalem (part II)}

\author{
Egzegeza patrystyczna J 4,21-24 \\ i prawdziwy kult w Jerozolimie (część II)
}

\begin{abstract}
We continue the presentation of reflection on Joh 4:21-24. (The first part of the study devoted to this theme appeared in "Biblica et Patristica Thoruniensia" 9(2016)4, p. 75-97). The Christian literature indicates that this passage allowed the development of various problems. The pericope in question had many interpretations in the history of exegesis. The writings of Christian authors have intertwined not only the questions of biblical interpretation. We have seen the authors of Antiochene provenance in the various historical-doctrinal contexts (John Chrysostom, Teodor of Mopsuestia and Teodoret of Cyrrus). Instead, on the other side, Cyril of Alexandria was found. Augustin of Hippo is approached to the Eastern Fathers. The use of the terms present in Joh 4:21-24 shows that this text was able to catalyze issues of decisive importance. It was not only exegetical or ritual problems, but also philosophical, historical and spiritual questions. Commentators of this period pointed to the cult "in the spirit and truth" as a characteristic feature of Christianity. However, the early Christian writers also saw the specific implications of this truth and its impact on the Christian's life.
\end{abstract}

Streszczenie. Przedstawiamy drugą część opracowania dotyczącego refleksji patrystycznej nad J 4,21-14. (Pierwsza część opracowania poświęconego temu tematowi pojawiła się w „Biblica et Patristica Thoruniensia” 9(2016)4, s. 75-97). Literatura chrześcijańska wskazuje, że ten fragment Janowy pozwolił na rozwinięcie problematyki o różnym charakterze. $Z$ tego względu nie brak różnic w sposobie interpretacji. Użycie terminologii i wyrażeń obecnych w analizowanej perykopie wskazuje, że ten tekst był swego rodzaju katalizatorem wielu kluczowych kwestii. Chodziło nie tylko o problemy egzegetyczne czy natury rytualnej, lecz także o kwestie filozoficzne, historyczne czy duchowe. W artykule wzięto pod uwagę autorów z kręgu antiocheńskiego (Jan Chryzostom, Teodor z Mopsuesti, Teodoret z Cyru), działających w różnych kontekstach 
historyczno-doktrynalnych. Przedstawicielem egzegezy aleksandryjskiej jest z kolei Cyryl z Aleksandrii. Do Ojców greckich w komentowaniu Ewangelii Janowej dołączył św. Augustyn. Komentatorzy epoki starożytnej wskazali na kult „w duchu i prawdzie” jako cechę charakterystyczną chrześcijaństwa. Autorzy wczesnochrześcijańscy dostrzegali jednak także konkretne implikacje tej prawdy i jej wpływ na życie chrześcijanina.

Key words: Gospel (of St. John); patristic exegesis; worship (spiritual); Jerusalem (temple of).

Słowa kluczowe: Ewangelia (św. Jana); egzegeza patrystyczna; kult (duchowy); Jerozolima (świątynia).

$\mathrm{I}_{\mathrm{in}}$ problemi interpretativi generati dall'esegesi di Gv 4,23-24 erano di vitale importanza e di notevole densità ${ }^{1}$. La seconda parte di questo studio cerca di completare il quadro di riflessione biblico-patristica sul culto spirituale e sul ruolo di Gerusalemme in esso.

Nella prima parte si è dovuti fermare con più attenzione su Origene. Il suo influsso sui Padri Cappadoci (Basilio e due Gregori: Nazianzeno e Nisseno), sembra fuori discussione ${ }^{2}$. Le interpretazioni dei Cappadoci di Gv 4,21-24 si focalizzano non nei tratti comuni ma piuttosto nelle reciproche differenze e nei diversi punti di vista. La linea della loro riflessione può essere tracciata dagli schemi che non vanno oltre la visione tradizionale dell'immagine di Gerusalemme e del culto (Basilio Magno), ad eccezione degli spunti teologici, presenti anche in Gregorio di Nazianzo per finire poi con un variopinto mosaico ermeneutico del Nisseno. Tuttavia queste prospettive hanno una caratteristica comune: il punto di gravità si sposta dalle sole questioni esegetiche ai problemi di tenore teologico-spirituale e dogmatico.

\section{Esegesi antiochena di Gv 4,21-24}

I commentari greci a Gv del IV-V secolo risentono del clima delle controversie cristologiche. In chiave antiariana si pone il commentario di Teodoro di Mopsuestia. L'altra opera di questo genere dell'ambiente antiocheno è quella di

1 La prima parte dello studio consacrato a questo tema è apparsa in „Biblica et Patristica Thoruniensia" 9/4 (2016), p. 75-97.

2 Lo dimostra il fatto che Basilio il Grande insieme a Gregorio Nazianzeno, composero una raccolta qualificata dei testi origeniani, la Filocalia. A questo proposito cfr. J. Gribomont, Lorigenisme de Saint Basile, p. 282-285. 
Giovanni Crisostomo. Invece agli inizi del V secolo viene preparato un ampio commento ad opera di Cirillo, vescovo di Alessandria d'Egitto.

Per S. Giovanni Crisostomo lo Spirito crea il popolo di Dio che è la Chiesa e opera nel suo tempo. In questa prospettiva si inserisce la constatazione che il Crisostomo fa sul passaggio dai Giudei e Samaritani ai cristiani.

„I veri adoratori adoreranno il Padre in spirito e verità" (Gv 4,23). Dicendo „i veri” [il Signore] respinge i Giudei insieme ai Samaritani. Se infatti i Giudei sono migliori dei Samaritani, sono però di gran lunga inferiori a coloro che verranno di quanto l'immagine è inferiore alla verità. Parla poi della Chiesa, perché essa stessa costituisce la vera e conveniente forma di adorazione di Dio. „Poiché il Padre cerca tali adoratori" (Gv 4,23). Dunque se egli li cercava tali, non perché pretendesse da loro proprio questo, ma perché concedeva ad essi tale maniera di adorarlo per benevolenza, affinché anchessi si avviassero verso la verità, chi sono dunque i veri adoratori? Quelli che non circoscrivono in un solo luogo il culto e adorano Dio in Spirito ${ }^{3}$.

Lo Spirito è il dono storico e interiore della salvezza. Egli rivela la natura di Dio, come il Figlio ne ha rivelato il volto misericordioso.

Quando Cristo dice: „Dio è Spirito” (Gv 4,24) non vuole intendere se non che Dio è incorporeo. È pertanto necessario che il culto dell'Incorporeo sia pure esso tale e che sia tributato a Dio per mezzo di ciò che vi è in noi d'incorporeo, cioè per mezzo dell'anima e della purezza della mente ${ }^{4}$.

Giovanni Crisostomo nel citare il testo della risposta di Gesù alla Samaritana, non ne propone l'esegesi particolareggiata, ma la suppone. Egli usa il testo evangelico nel suo significato letterale, servendosi della parafrasi. Da ciò risulta che nell'esercizio del culto divino è necessario distaccarsi sempre di più dagli aspetti sensibili che lo condizionano ${ }^{5}$ e lasciarsi innalzare dallo Spirito e da Cristo - verità verso il Padre.

Teodoro di Mopsuestia è indubbiamente un rappresentante assai caratteristico della scuola esegetica di Antiochia ${ }^{6}$. Tra suoi scritti sul Nuovo Testamento

3 Hom. in Joannem 33,2;2,39,

4 Hom. in Joannem 33,2; 2,40, .

5 Crisostomo afferma che al tempo dell'Antico Testamento Gerusalemme fu „la maestra di tutta la terra"; In Psalmum 47,1, PG 55, 217.

6 Egli fu altamente stimato dai suoi contemporanei ma condannato come eretico dopo la morte, condividendo la sorte del suo maestro - Diodoro di Tarso. La Chiesa nestoriana venera Teodoro come il grande interprete delle Scritture, non uguagliato da nessun altro. 
cè anche il commento al Vangelo di S. Giovanni ${ }^{7}$. Il IV Vangelo riveste per lui non solo l'importanza teologica, ma in generale costituisce un completamento di narrazioni sinottiche ${ }^{8}$. L'esegesi del Mopsuestiano si sviluppa nel contesto della controversia ariana. Egli accentuava fortemente il fatto dell'incarnazione ed è proprio che da questo concetto Teodoro tira le dovute conseguenze in ambito esegetico.

Teodoro di Mopsuestia mette la pericope di Gv 4 in confronto con i sinottici, sottolineando che nei loro scritti manca l'episodio giovanneo, complementare delle altre narrazioni ${ }^{9}$. Inoltre Giovanni ha confermato l'ordine storico dei discorsi, sostiene l'Antiocheno ${ }^{10}$. Si vede qui la sua preoccupazione filologico letteraria.

Teodoro afferma che Cristo, cioè l'Incarnato, ha fatto conoscere la dottrina cristiana dopo il paganesimo e il giudaismo. Il messaggio del Salvatore si rivela del tutto opposto al politeismo e complementare allebraismo. Quest'ultimo conosceva l'unico Dio ma non nella sua articolazione trinitaria. Il culmine della rivelazione è la persona di Cristo che parlava per „la potenza dello Spirito" Il vescovo di Mopsuestia fa un duplice discorso: cristologico e soteriologico.

Il Mopsuestiano evidenzia l'opposto comportamento dei Giudei e dei Samaritani innanzi al Signore. Ai Samaritani „fu sufficiente il semplice passaggio del Signore per conoscere la verità, cosa che i Giudei non furono capaci [di fare] neppure con la sua quotidiana permanenza fra loro" ${ }^{\prime 2}$.

Quegli spunti sono presenti nel seguito della sua interpretazione, costituendo una specifica chiave di lettura della pericope.

Perché non sembri che i Giudei e i Samaritani vadano equiparati, giacché predisse la fine di entrambi i santuari... Poi aggiunge: „Perché la salvezza proviene dai Giudei”. Non disse „nei Giudei”, ma „dai Giudei”. Perché, infatti, la salvezza non fu in essi, ma [provenne] da essi; perché da essi fu il Cristo nella carne. Pertanto, dice, la verità è „presso i Giudei”; e tuttavia entrambi [i santuari] saranno vanificati ${ }^{13}$.

7 Esso è pervenuto integralmente in una versione siriaca pubblicata da Vosté con una traduzione latina nel 1940. Cfr. J. M. Vosté, Theodori Mopsuesteni Commentarius in evangelium lohannis Apostoli. I frammenti greci sono pubblicati in PG 66, 728-785.

8 Cfr. L. Fatica, I Commentari a Giovanni di Teodoro di Mopsuestia e di Cirillo di Alessandria, p. 70.

9 Cfr. Commentarius in evangelium Johannis ap. II: 4,1.

10 Cfr. L. Fatica, I Commentari a Giovanni, p. 56.

11 Cfr. Commentarius in evangelium Johannis ap. II: 4,13-14.

12 Ibidem II: 4,3, CSCO 115, 60-61; cfr. L. Fatica, I Commentari a Giovanni, p. 57.

13 Commentarius in evangelium Johannis ap. II: 4,22, CSCO 115, 64. 
L'Autore rileva ulteriormente la contrapposizione Giudei/Samaritani già insita nel testo. A ciò si aggiunge il fatto che l'AT era ritenuto da Teodoro l'espressione della religione giudaica e tutto chiuso in un rigido monoteismo ${ }^{14}$, che lo caratterizza di fronte non solo al paganesimo ma anche al cristianesimo.

„Tempo verrà, anzi è già spuntato" (Gv 4,23), in cui Dio sarà adorato comè dovuto e conveniente alla sua natura. Infatti Dio è di natura incorporea, né è circoscritto da luoghi, ma è dovunque, e secondo tale nozione bisogna che sia adorato. Questi è il vero adoratore, colui che con il dovuto zelo gli tributa onore, e in coscienza limpida crede di poter dovunque parlare con l'Immenso. Pure non bisogna discutere come se soltanto qui o lì sia Dio e per questo vada adorato solo in un luogo; una tale questione è per Dio più offensiva che gratificante e avversa al giusto sentire degli adoratori ${ }^{15}$.

L'Antiocheno parte da una osservazione circa la natura di Dio. Da ciò scaturisce il carattere dell'adorazione rivolta al Signore: la forma di culto deve essere adeguata alla natura divina che è spirituale. La venuta di Cristo inizia una nuova fase, caratterizzata dalleconomia dello Spirito e della grazia, nettamente distinta dalla tappa precedente, caratterizzata dalleconomia della Legge. Essa però è tutta orientata verso il momento della consumazione finale di cui essa rappresenta l'anticipo e la promessa ${ }^{16}$. La promessa è presente nell'esegesi di Gv 4,23-24.

Teodoro segue fedelmente dettato biblico. Secondo lui esso si articola in due momenti: una costatazione teologica di carattere iniziale e poi le indicazioni per il culto umano, in pura coscienza.

Teodoro di Mopsuestia è convinto che le parole del Signore (Gv 4,21) e del Sl 136,4 non possono essere armonizzate. Per questo motivo manca la spiegazione tipologica della dellespressione „da Sion uscirà la Legge e la parola del Signore da Gerusalemme" (cfr. Mi 4,2 [variante di Is 2,3]) ${ }^{17}$. Anche se il versetto giovanneo indica che si tratta "del simbolo delle cose che si riferiscono a Cristo Signore”, tuttavia l'intento dell'Evangelista era quello di rappresentare „un modo di culto e non il luogo"18.

14 Egli si esprime nella maniera positiva sul culto giudaico in: Commentarius in $\mathrm{Mi}$ chaeam (IV,1-3) e in Zachariam (II,8-12); cfr. R. Devreesse, Essai sur Théodore de Mopsueste (Studi e Testi 141), p. 239.

15 Commentarius in evangelium Johannis ap. II: 4,24, CSCO 115, 64-65.

16 Cfr. ibidem III: 6, 2 a proposito di Gv 4, 13-14.

17 Commentarius in evangelium Johannis ap. II: 4,1-3, CSCO 115, 60.

18 Ibidem. 
Le stesse vicende storiche di Gerusalemme bastano per insegnare ai vari popoli le disposizioni divine. "Attraverso ciò che è capitato [a loro] riconosceranno la forza divina e stabiliranno la legge; perché per l'eternità possano a scendere a Gerusalemme e là adorare Dio nel modo giusto"19. Con l'evocare di Gv 4,21 ${ }^{20}$, Teodoro indica che è Gesù stesso che rigettò l'opinione di coloro che „limitavano il culto [divino] a sola Gerusalemme”21. Il testo evangelico, che il Mopsuestiano incorporò nella sua interpretazione delle profezie di Malachia, indica che

dopo che cessò a Gerusalemme ogni offerta secondo la Legge, ma anche arrivò la fine di possedere questo luogo, cominciò inesprimibile e spirituale offerta, che [noi] facciamo non solo nella città di Gerusalemme, ma in tutto lorbe terrestre dove cè fede. [Facciamolo] in memoria della passione e della risurrezione del Signore $^{22}$.

La tendenza generale di Teodoro è quella di ridurre gli elementi cristologici nell'interpretazione dei testi profetici. Questo è un altro indizio che la direzione della sua esegesi è opposta a quella tradizionale, condotta dagli autori di quel periodo ${ }^{23}$. In Teodoro manca la questione dell'attribuzione della formula „spirito e verità" allo Spirito Santo e a Cristo.

Questa serie di considerazioni può essere in linea di massima estesa anche ad altri esponenti della tradizione antiochena, soprattutto quella più legata a un'impostazione cristologica divisiva. L'esito dottrinale in Teodoro è stato determinato dalla forte tensione escatologica che pervade tutta la sua opera.

Nel caso di Teodoreto di Cirro si tratta di un lettore assiduo della Sacra Scrittura e dell'esegeta fortemente inserito nella tradizione esegetica antiochena. Egli ammette che ha commentato "tutti i profeti, il Salterio è l'Apostolo [Paolo]"24. È un'opera considerevole, se si tine conto che si tratti di un gerarca che doveva occuparsi di una Chiesa diffusa sul territorio molto vasto. Teodoreto commentò sistematicamente la Bibbia, servendosi di diversi generi letterari,

19 Commentarius in Zachariam XIV,12-19, PG 66, 593 C.

20 Nel commentario a Gv non si è conservata la sua esegesi; cfr. Commentarius in evangelium Johannis ap. II: 4,9.

21 Commentarius in Malachiam III,3.4, PG 66, 624 A.

22 Ibidem.

23 L'AT è considerato come un'unità chiusa, che ha solo parzialmente un ruolo di preannunzio profetico e simbolico della Nuova Alleanza. Cfr. M. Simonetti, Lettera e/o allegoria. Un contributo alla storia dell'esegesi patristica, p. 169; 170.

24 Epistula 82, SCh 98, 202-203. Si veda G.W. Ashbyl, Theodoret of Cyrrhus as Exegete of the Old Testament. 
per dare un fondamento scritturistico alla dottrina cristologica. Pur professando difisismo, non è arrivato allestremismo di Nestorio ${ }^{25}$. La causa di ciò sta nel fatto che egli si è occupato soprattutto dell'esegesi dell'Antico Testamento, principalmente dei libri profetici. Teodoreto non possiede un commento sistematico a Giovanni. Egli semplicemente evoca le espressioni scelte di questo Evangelista. Egli impiega Gv 4,24 nei contesti dottrinali ${ }^{26}$, perché si tratta di un versetto che indica la natura spirituale di $\mathrm{Dio}^{27}$.

Il vescovo di Cirro non converge con un altro Antiocheno che era Teodoro di Mopsuestia. Quest'ultimo infatti vedeva il contrasto tra il monte del Signore e il culto di Dio „in spirito e verità". Secondo l'interpretazione letterale le parole di Gv 4,21, escludono il monte di Sion. Teodoreto evita interpretazione troppo letterale ${ }^{28}$. Egli indica Gerusalemme come luogo da cui il Vangelo si è espanso tra tutti i popoli ${ }^{29}$. Ciò è chiaro indizio che, alle volte, il vescovo di Cirro non poteva fare a meno dalle acquisizioni dell'esegesi origeniana, mostrandosi però libero nello sfruttare poi i suoi vari elementi.

Un intreccio tra gli elementi concreti e figurativi si vede spesso nel trattare la questione di Gerusalemme da parte di questo scrittore. Secondo la visione del vescovo di Cirro la capitale della Giudea è ormai un centro cristiano. Il culto veterotestamentario è da considerare un capitolo chiuso. I figli della Chiesa „accorrono da tutto il mondo verso Gerusalemme non per adorare Dio nel tempio giudaico, ma per vedere i famosi luoghi della passione, della risurrezione e dell'ascensione [del Signore]"30. Il vescovo di Cirro indica con chiarezza che cè un fattore decisivo: Gerusalemme è diventata la metropoli dei credenti in Cristo $^{31}$. Il metropolita siriano sottolinea che la città santa si è trovata in mezzo ai popoli perché „[essi] imparassero ogni pietà e il vero culto [di Dio]”32. Da quel

25 Cfr. J.-N. Guinot, Léveque exégète: Théodoret de Cyr, p. 335-359.

26 Cfr. due brevi allusioni in Eranistes I e II.

27 Cfr. Eranistes II; si veda G.H. Ettinger, Theodoret of Cyrus. Eranistes, p. 169.

28 Nell'AT l'esistenza di un tempio non limitava Dio, ma si riferiva al luogo della sua rivelazione; Interpretatio in Ps. 134,21; PG 80, col. 1920 C.

29 Si veda M. Simonetti, Lettera e/o allegoria, p. 195. Cfr. Interpretatio in Ps. 116,1.

30 Commentaria in Is. XIX,60,4, SCh 315, p. 244-245.

31 „Le parole [...] ci ammaestrano: «Tu riconoscerai che io sono il Signore, che coloro che sperano in me non saranno delusi» (Is 49,23). Ogni giorno vediamo che ciò avviene nelle chiese... Si può vedere il compimento di [questa] profezia in modo particolare a Gerusalemme"; Commentaria in Is. XV: 49, 21-23, SCh 315, p. 94-97.

32 Explanatio in Ez. II,5,5-6, PG 81, 863 B-C. 
luogo „per tutti sono sgorgate le fonti della salvezza” ${ }^{33}$. Si tratta, quindi, del trionfo del sacrificio mistico e del culto reso a Dio vero ${ }^{34}$.

La novità dell'esegesi di Teodoreto consiste dunque nella rivendicazione della lettura cristologica del testo sacro, contro il riduzionismo di Diodoro e Teodoro di Mopsuestia. D'altro canto l'atteggiamento del vescovo di Cirro spiega bene l'atmosfera creatasi nel seno dell'esegesi cristiana. La spiritualizzazione del culto è passata al secondo piano: erano le testimonianze materiali e concrete a rivelare la verità dell'adorazione resa dai cristiani al Signore.

La prospettiva delineata dalla riflessione del vescovo siriano non è tanto lontana dal punto di vista rappresentato da S. Girolamo. Prescindendo dalle differenze sostanziali tra i due autori (del resto ovvie), come la lingua, l'ambiente, l'indirizzo teologico e il momento storico, si possono accordare molte somiglianze. Infatti abbiamo a che fare con la convinzione precisa dell'eccezionalità del culto situato nei luoghi santi.

Girolamo si presenta principalmente come uno studioso di Bibbia. La sua attività di traduttore e interprete delle Scritture lo mette in relazione con Origene. Alla pari di Teodoreto, il Dalmata offre una dimostrazione eloquente di un'esegesi contestuale, a motivo del suo particolare attaccamento ai luoghi san$\mathrm{ti}^{35}$. Il Dalmata era convinto fino in fondo che si può ritenere „di non poter raggiungere la perfezione” senza adorare Cristo ,in quei luoghi dove il Vangelo, prima che altrove, aveva irradiato il suo splendore dalla Croce" ${ }^{\prime 6}$. Nel caso del monaco di Betlemme si possono fare solo le considerazioni sommarie, ma che indicano atmosfera in cui furono analizzati alcuni testi biblici. Così l'esegeta latino fa menzione di coloro che „alzano le mani pure [verso Dio] in ogni luogo"37. Si ha qui l'idea del culto spirituale, espressa però alla maniera un pò enigmatica $^{38}$. In questo contesto il IV Vangelo non ha avuto un rilievo particolare. La traduzione geronimiana differenzia dalle versioni precedenti del

33 Historia religiosa IX,2, SCh 234, p. 408-409.

34 Cfr. J.-N. Guinot, Lexégèse de Théodoret de Cyr, p. 469.

35 A questo proposito si veda M.C. Paczkowski, Gerusalemme in Origene e San Girolamo, p. 115-123; L. Perrone, „The Mystery of Judaea” (Jerome, Ep. 46): The Holy City of Jerusalem between History and Symbol in Early Christian Thought, in: L.I. Levine (ed.), Jerusalem. Its Sanctity and Centrality to Judaism, Christianity and Islam, p. 221-239.

36 Epistula 47,9, Labourt, Saint Jérôme. Lettres, II, p. 109.

37 In Esaiam XVIII,66,24, CCL 73 A, p. 797.

38 Già Lattanzio sottolineava la necessità del culto spirituale; cfr. Epitome institutionum divinarum LIII. 
„Vangelo spirituale" 39 . È una nota interessante, ma non pertinente al tema di questo studio.

\section{Interpretazione di Cirillo Alessandrino}

La vera e propria cesura dello sviluppo della rivoluzionaria interpretazione origeniana di Gv 4,23-24 costituisce l'atteggiamento di Cirillo d'Alessandriaa ${ }^{40}$. I commentari al IV Vangelo composti da Giovanni Crisostomo e Teodoro di Mopsuestia erano a lui sconosciuti. Bisogna notare però che malgrado il fatto che egli abbia composto unopera intitolata Dell'adorazione e il culto in spirito e verità, ove il Pentateuco viene reinterpretato in chiave tipologico-cristologica, il metropolita egiziano dedica in maniera assolutamente sorprendente pochissimo spazio. Si tratta di Gv 4,24, del quale egli riporta soltanto due mere citazioni, prive di qualsiasi commento ${ }^{41}$. Questo sostanziale disinteresse è confermato da un esame del suo Commento a Giovanni ${ }^{42}$. Questa opera è stata composta tra il 425-428, cioè poco prima del concilio di Efeso (431). Il criterio ermeneutico è costituito dal Simbolo niceno-costantinopolitano (non è ancora scoppiata la polemica con Nestorio). La lettura di Gv risulta piuttosto letterale ed è indirizzata per contestare diverse tesi ereticali ${ }^{43}$. Esse sono d'interesse non indifferente, perché fanno luce sui marginali ambienti cristiani dell'inizio del $\mathrm{V}$ secolo ${ }^{44}$. Dalle polemiche condotte da Cirillo emergono le convinzioni origeniste degli eterodossi, che erano popolari negli ambienti monastici. Da qui questa notevole sobrietà del metropolita nel commentare il Vangelo spirituale.

39 Lesempio del genere si può trovare in Tertulliano che riporta Gv 4,23 in: De oratione 28,2. Ne fa fede poi il testo di Gv 4,23 citato da Agostino. Le citazioni agostiniane hanno il tempo futuro, che è assente nella Volgata. Cfr. H. Houghton, Augustine's Text of John. Patristic Citations and Latin Gospel Manuscripts, p. 225-226.

40 Cfr. G. Lettieri, In spirito e/o verità. Da Origene a Tommaso d'Aquino, in: P.C. Bori (a cura di), In spirito e verità. Lettura di Giovanni 4,23-24, p. 69-70.

41 Cfr. De adoratione et cultu in spiritu et veritate I; IX; in: PG 68,136 A; 589 C. Si veda N. Russell, Cyril of Alexandria, p. 13.

42 Alcuni lo ritengono il più antico commento esegetico di Cirillo. Questa voluminosa opera comprende dodici libri suddivisi in capitoli. I libri 7 e 8, che interpretano Gv 10,18-22, 48, sono scomparsi, tranne un piccolo numero di frammenti conservati nelle catene, e di autenticità peraltro dubbia. A proposito di questopera cfr. N. Russell, Cyril of Alexandria, p. 96-97.

43 Sul fatto delle eresie cfr. ad es. In Isaiam II,2; 14,1-3; III,4; 37,36-38.

44 Cfr. M. Cristiani, Il Prologo di Giovanni da Agostino a Tommaso d'Aquino, Annali di storia dell'esegesi 11/1 (1994), p. 52-53. 
Bisogna notare che Giovanni Crisostomo e Teodoro di Mopsuestia hanno redatto i loro commentari in prospettiva antiariana sfruttando la portata cristologica del Vangelo spirituale.

Il commento a Gv 4,21 costituisce il passaggio al capitolo V del libro secondo di questa opera cirilliana, dove il Figlio è definito oggetto di adorazione con il Padre. Lamorevole umiltà del Figlio di Dio dona agli uomini un esempio supremo ${ }^{45}$.

[Egli] indica che è presente il tempo della sua venuta, ma dice che i simboli si trasformeranno in realtà, e l'ombra della Legge in culto spirituale. [Il Signore] dice che il vero adoratore, cioè uomo spirituale, sarà portato per mezzo dell'insegnamento evangelico, alla vita gradita al Padre, e correrà, in qualche modo, con maggiore prontezza verso la familiarità di con $\mathrm{Dio}^{46}$.

Cirillo presenta un'interpretazione affrettata e poco originale di Gv 4,23. Ripropone, in termini origeniani, distinzione tra lettera della Legge e interiorità del culto spirituale, tra i simboli materiali, ormai superati e la realtà cristiana, capace di comprendere l'immateriale onnipresenza di $\mathrm{Dio}^{47}$. Il metropolita egiziano lo fa nellesortazione di carattere morale:

Infatti „Dio è spirito” (Gv 4,24), se è paragonato con la natura corporea, e perciò giustamente è adoratore spirituale non colui il quale, alla maniera giudaica, offre una pietà figurativamente e tipicamente, ma colui che risplende evangelicamente di opere buone derivanti dalle virtù, e compie la vera adorazione secondo i retti dettami dei divini insegnamenti ${ }^{48}$.

Il nuovo culto culmina, quindi, nell'ascesa spirituale sino alla suprema perfezione evangelica che è sostenuta dall'azione illuminatrice dello Spirito Santo ${ }^{49}$. Essa è in netto contrasto con la vecchia economia.

Considera come si realizza nelladorazione il tipo del culto nello Spirito, al quale sono condotte le genti attraverso la fede. Infatti era uso del popolo d'Israele di venerare Dio con immolazione di tori e di altri animali e con incensi secondo il precetto della Legge. Coloro invece che tra i pagani sono venuti alla fede [e] non que-

\footnotetext{
45 Cfr. Commentarii in Joannem II, 5, in riferimento a Gv 4,22.

46 Ibidem II, 5 (4,23-24), PG 73, 313 B-C.

47 Cfr. Commentarii in Joannem II, 4.

48 Ibidem II, 5 (4,23-24), PG 73, 313 B.

49 Cfr. Commentarii in Joannem VI $(8,46) ; \mathrm{X}, 2$ (15,3); In Isaiam IV, 1. Cirillo si riferisce
} a Gv 4,24. 
sta, ma unaltra strada hanno preso per dare culto a Dio, una via spirituale che Dio stesso dice essergli molto più gradita e cara $[\ldots]$. Gesù Cristo stesso nostro Signore, mostrando che l’adorazione spirituale è migliore di quella legale (segue la citazione di Gv 4,21.23-24) ${ }^{50}$.

Cirillo tocca il tema riguardante la costruzione del tempio di Dio: luogo della sua presenza e sua „abitazione”. Il tema viene esplicitamente congiunto con il culto spirituale. Il tempio di Dio sarà quell'anima in cui si compie il vero culto e l'adorazione conveniente al Signore ${ }^{51}$.

Cirillo di Alessandria elenca nel modo particolare varie istituzioni dell'Antico Testamento e del tempio gerosolimitano ${ }^{52}$. Si deve però prendere in considerazione che la Chiesa non fu chiamata „casa delle offerte e dell'incenso", come il tempio giudaico, ma si tratta della preghiera cioè del „culto spirituale" 53 . Tuttavia il metropolita egiziano lascia a parte gli aspetti del culto „in spirito e verità", concentrandosi sulla descrizione del santuario anticotestamentario. Il tempio salomonico è legato al periodo della „fede semplice” d'Israele e della convinzione erronea del Sion come dimora divina ${ }^{54}$.

Il metropolita egiziano lo esprimeva commentando testo di Gv 4,22, in cui faceva leva sulle differenze tra le concezioni di culto dei Samaritani e degli Ebrei.

[I primi] adorano Dio senza capire e approfondire; i Giudei, invece, per avere conosciuto, attraverso la Legge e i profeti ${ }^{55}$, per quanto è stato loro possibile, il vero Dio, si trovano in una condizione di superiorità. Perciò [il Signore] dice che i Samaritani non sanno, mentre i Giudei lo sanno... [Egli] colloca se stesso tra gli adoratori, in quanto uomo, Egli che con Dio Padre è adorato, sia da noi che dai santi Angeli ${ }^{56}$.

Il commento Gv 4,22 è strettamente teologico e prende avvio dall'affermazione secondo cui Cristo si è espresso nel modo chiaro parlando della sua umanità ${ }^{57}$. Sorprende il fatto che il metropolita egiziano dà tanta importanza alla

50 Commentarii in Joannem VI (9,38), PG 73, 1012 C-D - 1013 A.

51 Cirillo si riferisce ad Origene; cfr. In exodum hom. IX, 4-5.

52 Israele viene paragonato alla vigna; cfr. In Isaiam I, 3; 5, 3.

53 Ibidem V,4;60,4-7, PG 70, 1329 C-D.

54 Cfr. ad es. Contra Julianum 2.

55 Questa espressione indica tutto l'Antico Testamento.

56 Commentarii in Joannem II,5 (4,22), PG 73, 304 C-D.

57 Con aiuto di Fil 2,7.8 Cirillo afferma che Cristo, pur umiliatosi nella nostra umanità, è rimasto nella gloria del Padre. Colui che si è umiliato rimane presso il Padre. Il plurale 
venerazione del Padre da parte di Cristo. Questo atteggiamento mostrato dal Figlio è segno del umiltà del Signore, non della sua inferiorità. Esso però indica anche il fatto dell'assunzione piena della natura umana ${ }^{58}$.

Il metropolita alessandrino si serve poi dell'immagine dell'offerta in moneta imposta dalla Legge antica (siclo, cfr. Es 30,11-13) per affermare che il culto legale è stato annullato. „Noi riteniamo che il Signore dell'universo non debba più essere onorato con doni esterni... Ma poiché siamo veri adoratori, noi adoriamo Dio Padre in spirito e verità. Bisogna pensare che nel senso letterale della Legge sia nascosto questo insegnamento" 59 .

Altre riflessioni cirilliane che mettono in risalto la interdipendenza tra l'adorazione spirituale e perfezione morale ${ }^{60}$.

Laffermazione di Cristo che i veri adoratori avrebbero offerto a Dio Padre il culto spirituale implica anche la speranza messianica. Cirillo dichiara che „la donna [samaritana] subito vola col pensiero alla speranza divulgata dai Giudei, elevandosi ai pensieri superiori alla sua mente" 61 .

Il metropolita alessandrino insiste in riferimento a Gv 4,23-24 sull'identificazione giovannea tra lo Spirito Santo e l'Amore divino (cfr. $1 \mathrm{Gv} 4$ ) che accende il desiderio di Dio. La donna [samaritana] comprese che „era... già presente il tempo, nel quale i veri adoratori non avrebbero adorato più sui monti della terra, ma avrebbero offerto a Dio Padre un culto più sublime e spirituale"62.

La venuta del Salvatore indica instaurazione di un nuovo tempio, molto più glorioso dell'antico. Per Cirillo lo indica il testo profetico di Aggeo. Il vescovo di Alessandria scrive:

Al tempo della venuta del nostro Salvatore apparve un tempio divino senza alcun confronto più glorioso, più splendido ed eccellente di quello antico. Quanto superiore era la religione di Cristo e del Vangelo al culto dell'antica Legge e quanto superiore è la realtà in confronto alla sua ombra, tanto più nobile è il tempio nuovo rispetto all'antico. Penso che si possa aggiungere anche un'altra cosa. Il tempio era unico, quello di Gerusalemme, e il solo popolo d'Israele offriva in esso i suoi

„noi adoriamo” è indizio dell'adeguamento del Verbo alla condizione assunta attraverso l'Incarnazione. Il metropolita di Alessandria afferma che il Salvatore „necessitava piuttosto dire: «Io adoro ciò che conosco»"; Commentarii in Joannem II,5 (4,22), PG 73, 313 A.

58 Probabilmente Cirillo prende di mira gli apollinaristi.

59 Commentarii in Joannem II,5 (4,22), PG 73, 309 C. Il testo - chiave è costituito dalla pericope di Mt 17,24-27.

60 Cfr. Commentarii in Joannem II,5 (4,23). Si veda anche ibidem II,4 (4,10); IV,1 $(6,40)$.

61 Ibidem II,5 (4,25), PG 73, 313 C.

62 Ibidem II,5 (4,26), PG 73, 315 A-B. 
sacrifici. Ma dopo che l'Unigenito si fece simile a noi, pur essendo „Dio e Signore, nostra luce" (Sl 117,27), come dice la Scrittura, il mondo intero si è riempito di sacri edifici e d'innumerevoli adoratori che onorano il Dio dell'universo con sacrifici ed incensi spirituali ${ }^{63}$.

Riferendosi a Ml 1,11 il metropolita d'Egitto sostiene che

la gloria dell'ultimo tempio, cioè della Chiesa, sarebbe stata più grande. A quanti lavoravano con impegno e fatica alla sua edificazione, sarà dato dal Salvatore come dono e regalo; celeste Cristo, che è la pace di tutti ${ }^{64}$. Noi allora, per mezzo di lui, potremo presentarci al Padre in un solo Spirito (cfr. Ef 2,18) ${ }^{65}$.

Nonostante alcuni interessanti suggerimenti interpretativi, Cirillo non dimostra un particolare interesse di fronte alle parole di Gesù pronunciate al pozzo di Giacobbe. Il brano evangelico risultava a lui troppo eversivo, capace di mettere in crisi, forse anche di relativizzare il sistema religioso cristiano, che nella sua storica visibilità ha soppiantato quello giudaico. Cirillo si preoccupa infatti di notare come l'interiore culto cristiano anziché rimanere nascosto nell'intimità del credente, risplenda visibilmente. Il cristianesimo doveva essere visto come culto che offuscò gli splendori della religione pagana in Egitto. Poi il cristiano adora Dio ubbidendo ai „dettami dei dogmi divini” che non possono essere trascurati. Si tratta di un culto totalmente differente perché „tutto è stato rinnovato in Cristo: il culto, la vita e la legislazione. Noi non abbiamo aderito alle ombre oppure agli inutili simboli [tipi]. A Dio, che è al di sopra di tutto, noi offriamo adorazione e culto in spirito e verità (cfr. Gv 4,23)"66.

In Cirillo si scorge che è del tutto trascurato il ruolo dello Spirito Santo nell'atto dell'adorazione, come anche la valenza trinitaria di Gv 4,2367. Complementare sotto questo aspetto appare la riflessione di Agostino d'Ippona che si cercherà di delineare in seguito.

63 In Aggaeum 14, PG 71, 1018 D.

64 Sul tema Cristo - pace cfr. In Isaiam V,6; 66,10-12.

65 In Aggaeum 14, PG 71, 1019 B.

66 In Isaiam V, 6, PG 70, 1437 C.

67 Cirillo però abbozza una dottrina dei nomi divini, donde emergono le relazioni intratrinitarie. Cfr. L. Fatica, I Commentari a Giovanni, p. 67. 


\section{Agostino di fronte a Gv 4,21-24}

Per S. Agostino la Sacra Scrittura costituisce la fonte principale della predicazione e della riflessione teologica. In questa angolatura egli evoca i fatti dell'Antico Testamento, come ad esempio le profezie. Gli antichi profeti ricordavano al popolo eletto la prospettiva del ritorno in patria e il rinnovo del culto nel tempio di Gerusalemme. I Salmi esprimono la nostalgia Israele per il tempio in rovina. Nella concezione dell'autore De civitate Dei questi fatti rappresentano la visione della schiavitù e della liberazione, della distruzione e della ricostruzione nella storia della Chiesa e dell'individuo.

Questa interpretazione non è differente dalle opinioni della maggioranza degli scrittori antichi che nella prospettiva simile riassumevano la storia del popolo d'Israele. La base è costituita dalla convinzione e del ruolo eccezionale che aveva la storia del popolo eletto e ciò è stato confermato dalla Nuova Alleanza $^{68}$. Per S. Agostino non si poteva fare meno di Israele e della storia della sua capitale con il santuario. Ciononostante secondo la sua opinione la Chiesa si è liberata dai „vincoli carnali” del particolarismo ebraico ${ }^{69}$.

A differenza degli altri scrittori dell'antichità cristiana Ipponate non si occupa alla maniera particolareggiata dei luoghi di culto, in particolare quelli a Gerusalemme. Si potrebbe supporre che per l'autore della De civitate Dei lo spazio sacro e il vivere la realtà dei luoghi santi non era l'argomento di grande interesse. Ciò fa riflettere tanto perché il vescovo di Ippona incoraggiava il culto dei martiri e faceva delle omelie su di loro, senza escludere i pellegrinaggio ai luoghi particolari. Però Agostino scelse il silenzio nella questione dei materiali luoghi di culto a Gerusalemme. Ciò è dovuto alla sua inclinazione intellettuale e il legame con la dottrina paolina della divina presenza nel mondo, come pure della prospettiva spirituale del tempio divino ${ }^{70}$.

68 Cfr. ad esempio De civitate Dei XV,26; XVII,16; XVIII,54. Vari testi annunciano che „Gerusalemme è la città di Dio e la predizione che in essa vi sarà la casa di Dio"; ibidem XVII,3, CCL 48, p. 553-554.

69 „Israele sono i figli della Chiesa. Quanto a Sion, fu una città [storica], ma questa fu abbattuta, anche se sopra le sue rovine seguitarono ad abitarci materialmente i santi. La Sion vera e la Gerusalemme vera... è la comunità dei santi, che ci ha allevati”. Enarrationes in Ps. 149,5, NBA 28, p. 902-903.

70 Ne è testimonianza l'interpretazione del Sl 131: „Tabernacolo o tenda di Dio è la Chiesa del tempo presente, casa di Dio invece la Chiesa celeste, la Gerusalemme verso la quale siamo incamminati”; Enarrationes in Ps. 131,10, NBA 28, p. 280-281. Cfr. anche De civitate Dei XX,11. 
Questo pensiero dell'Ipponate diventa più convincente se si prende in considerazione la tensione tra il tempo presente e l'eternità, la temporaneità del santuario gerosolimitano ${ }^{71}$ e la permanenza eterna della „casa di Dio” - la Gerusalemme celeste. La fine ultima è la gloria nelleternità ${ }^{72}$ e quindi, secondo l'opinione di Agostino, non è il gran privilegio in possesso di un luogo stabile di culto nella Gerusalemme terrena ${ }^{73}$.

Nonostante la presenza dei temi che riguardano Gerusalemme e il suo santuario, Agostino impiega Gv 4,23-24 quasi sempre in contesti dottrinalmente poco impegnativi. Lo fa a differenza di altre pericopi quando approda al testo del commento come all'esito maturo della propria attività esegetica e speculativa $^{74}$. Del resto, il commentario agostiniano, evidentemente condizionato dal carattere omiletico, costituisce una vera e propria lezione di ortodossia trinitaria $^{75}$, rivolta al popolo di Ippona. Il Doctor Gratiae invita a sollevarsi alla comprensione spirituale del testo ${ }^{76}$.

Tuttavia nel caso di Gv 4,23-24 si tratta di un testo che entrava nella polemica con i donatisti. Un esempio palese di questa esegesi è un sermone anonimo della collezione viennese (Sermones Escorial) ${ }^{77}$. Esso inizia con esortazione alla vita cristiana ${ }^{78}$. Lautore donatista distingue i „falsi credenti” (probabilmente appartenenti alla Chiesa cattolica) dai veri cristiani (della sua sètta). Lappartenenza alla fede donatista significa essere seguace della verità ${ }^{79}$. „Colui che adora Dio in spirito e verità" appartiene, secondo l'anonimo scrittore, ai donatisti. Un vero credente conduce in questa maniera una vita moralmente pura.

71 Agostino accenna che „Cristo amò la sua città e ne ebbe misericordia; da essa ordinò che prendesse inizio la sua predicazione: «Incominciando da Gerusalemme». Lì volle che si iniziasse a parlare del suo nome"; In I Joannis 2,3, SCh 75, p. 158.

72 „Prima enim domus, id est, cives terrenae Hierusalem”; Sermo 50,11, NBA 29, p. 958-959.

73 Enarrationes in Ps. 121,3, NBA 28, p. 6-7.

74 Sull'esegesi agostiniana di questo brano cfr. G. Lettieri, In spirito e/o verità, p. 62-65.

75 L'autore è convinto che l'adorazione autentica di Dio ha come principi che la attuano il mistero dello Spirito e del Figlio. Il vero culto ha quindi una struttura trinitaria: avviene nello Spirito e nel Figlio ed è diretto al Padre.

76 Cfr. Tractatus in Johannem I,1.

77 Su queste omelie cfr. J. L. Grabau, John 4:23-24 in North African Preaching, p. 138140.

78 Questa omelia nel Rinascimento venne attribuita a Giovanni Crisostomo. Si veda J. L. Grabau, John 4:23-24, p. 138.

79 Sermo Escorial 16, PLS 4, 706. 
Per la vera "Chiesa" si intende quindi il luogo degli eletti che obbediscono alla Legge divina ${ }^{80}$.

Sant'Agostino è assai è lontano da queste convinzioni e ciò rivelano la sua esegesi e l'ecclesiologia, se comparate con le idee donatiste ${ }^{81}$. La controversia con i donatisti si sviluppava intorno all'idea della Chiesa. Per Agostino è nel suo corpo unificato sta l'assenza della sua identità quale società universale e visibile, unione dei battezzati che credono. Non si tratta solo di una comunità terrena ${ }^{82}$.

Agostino dà a Gv 4,23-24 un'interpretazione assai particolareggiata, fortemente allegorizzante, con alcuni spunti originali. Questo modo di trattare il testo giovanneo ${ }^{83}$ è l'indizio di precise opzioni esegetiche dell'Ipponate. Lo costituisce il XV Tractatus (predicato nel 407) del commento a $\mathrm{Gv}^{84}$. Bisogna notare che questa parte dellopera agostiniana, pur conservando il carattere pastorale, è però ricca di contenuto teologico-filosofico e spirituale ${ }^{85}$. Egli, tra l'altro, evidenzia il suo disaccordo con il punto di vista donatista ${ }^{86}$. Nel caso di Gv 4,23 Agostino preferisce un approccio paolino a questo testo. Difendendo la vera Chiesa che esercita culto in spirito e verità, si richiama alla natura divina, collegando questa verità con Gv 4,24 („Dio è spirito”) ${ }^{87}$. Questo è il primo approccio agostiniano, perché dopo il nostro si occupa di Gv 4,22-23.

\footnotetext{
80 Cfr. ibidem, PLS 4, 707.

81 Lautore latino conosceva l'interpretazione donatista della Bibbia dalle opere di Ticonio.

82 H. Chadwick, The Church in Ancient Society: From Galilee to Gregory the Great, p. 391-392.

83 „[Cristo] domanda da bere e promette di dissetare. È bisognoso come uno che aspetta di ricevere, e abbonda come chi è in grado di saziare. «Se conoscessi, dice, il dono di Dio». Il dono di Dio è lo Spirito Santo. Ma Gesù parla alla donna in maniera ancora velata, e a poco a poco si apre una via al cuore di lei. Forse già la istruisce»?"; Tractatus in Johannem XV,16, NBA 24/1, p. 362-363.

84 Nella Chiesa occidentale latina il commento giovanneo del vescovo d'Ippona domina incontrastato. Esso si presenta come 124 discorsi, distinti in due gruppi (1-54 e 55-124). La data di composizione è molto discussa: 411/416, 413/418 o dopo il 419-420.

85 Cfr. J. Quasten, Patrologia, vol. 3, p. 374.

86 A proposito di battesimo e eucarestia; cfr. Tractatus in Johannem XV,8.

87 Cfr. Tractatus in Johannem XV,25. Si veda J. L. Grabau, John 4:23-24, p. 149.
} 
Per il vescovo di Ippona la Samaritana è la figura della Chiesa ${ }^{88}$ che viene dai non-giudei, ovvero dai gentili ${ }^{89}$. Gesù „stanco" rappresenta il Cristo incarnato, umiliatosi ${ }^{90}$. Agostino non esita a identificare il dono di Dio con lo Spirito Santo ${ }^{91}$ e segue con attenzione la finezza psicologica con la quale Gesù conduce la Samaritana alla conversione ${ }^{92}$.

L'Ipponate specifica che Cristo parla alla maniera spirituale e non carnale ${ }^{93}$, ma la Samaritana continua a interpretare le sue parole materialmente ${ }^{94}$. Agostino cercava in tal modo di sottolineare la gratuità del dono dello Spirito, non meritato dalla donna, attratta ancora soltanto dai piaceri mondani incapaci di appagare l'anima umana ${ }^{95}$.

Dopo questa introduzione, Agostino passa a trattare, pur se molto rapidamente, del luogo dell'adorazione di Dio, accomunando del tutto Samaritani e Giudei nellerrore, morale prima ancora che intellettuale, di pretendere di possedere come proprietà Dio stesso, tramite il possesso del luogo, il Tempio o il monte Garizim. Il vescovo di Ippona suppone che

[Era] in corso una discussione vivace tra i Samaritani e i Giudei, per il fatto che i Giudei adoravano Dio nel tempio costruito da Salomone, mentre i Samaritani, esclusi, non adoravano Dio in quel tempio. Perciò i Giudei si ritenevano migliori per il fatto che adoravano Dio nel tempio... Gli uni e gli altri contendevano tra loro, privi, gli uni e gli altri, della conoscenza di Dio perché non avevano marito: e si gonfiavano gli uni nei confronti degli altri, i Giudei per il tempio, i Samaritani per il monte [Garizim $]^{96}$.

88 „E arrivò una donna» (Gv 4,7): figura della Chiesa, non già giustificata, ma ormai sul punto di esserlo (forma Ecclesiae, non iam iustificatae, sed iam iustificandae)", Tractatus in Johannem XV,7, NBA 24/1, p. 350-351.

89 „I Samaritani non appartenevano al popolo giudeo: erano infatti degli stranieri. È significativo il fatto che questa donna, la quale era figura della Chiesa, provenisse da un popolo straniero. La Chiesa infatti sarebbe venuta dai pagani, che, per i giudei erano stranieri"; Tractatus in Johannem XV,10, NBA 24/1, p. 354-355.

90 Cfr. Tractatus in Johannem XV,6-8.

91 Secondo gli esegeti moderni l'espressione „in Spirito” significa l'ispirazione, la mozione, l'impulso dello Spirito di Dio nella esistenza di fede; cfr. G. Ferraro, Lo Spirito e Cristo nel quarto Vangelo, p. 99-100.

92 Cfr. Tractatus in Johannem XV,13.

93 Tractatus in Johannem XV,14, NBA 24/1, p. 358-359.

94 Cfr. Tractatus in Johannem XV,15.

95 Cfr. Tractatus in Johannem XV,16-17.

96 „Contendebant utrique ignari, quia virum non habentes; illi pro templo, illi pro monte inflabantur adversus invicem"; Tractatus in Johannem XV,23, NBA 24/1, p. 366-367. 
Appare qui l'interpretazione platonizzante del culto voluto da Dio (in spirito e verità) come adorazione interiore e invisibile ${ }^{97}$. Particolarmente interessante e ricca di potenziali sviluppi, è invece la nota sulla perdita dei privilegi da parte di Samaritani e Giudei che peccano per il superbo desiderio di eccellen$\mathrm{za}^{98}$. In tal senso, la pretesa di „adorare Dio sul monte” viene letta da Agostino come pretesa di egoistica autogratificazione ${ }^{99}$.

Lo adoreranno, non su questo monte, non nel tempio, ma in „spirito e verità" (Gv 4,24). „Il Padre, infatti, cerca tali adoratori” (Gv 4,23). Perché il Padre cerca chi lo adori, non sul monte, non nel tempio, ma in spirito e verità? Perché „Dio è spirito". Se Dio fosse corpo, sarebbe stato necessario adorarlo sul monte, perché il monte è corporeo; sarebbe stato necessario adorarlo nel tempio, perché il tempio è materiale. Invece, „Dio è spirito, e i suoi adoratori devono adorarlo in spirito e verità" $(G v 4,24)^{100}$.

Lo „spirito” e la „verità" vengono contrapposti ai antichi luoghi di culto (monte Garizim e Gerusalemme). "Adorare in spirito” significa che il culto a Dio Padre è reso possibile e ispirato dallo Spirito Santo come principio interiore di vita; veri adoratori sono rinati „da acqua e da Spirito” (Gv 3,5). Solo l'amore di Dio, accolto dall'umile ubbidienza dell'uomo, riunisce sia Samaritani (pagani) che Giudei ${ }^{101}$ nell'unica assemblea spirituale (Chiesa) ${ }^{102}$. Ciò è però unicamente dalleterna volontà di Dio, che Cristo realizza nel tempo tramite la grazia operante del suo Spirito, capace di chiamare, illuminare e attuare la fede nei suoi credenti ${ }^{103}$.

97 Cfr. Tractatus in Johannem XV,24; Sermo 16/A,9.

98 Agostino insiste non sullosservanza esteriore dei precetti, ma su quella interiore. Invece in Pelagio c'era un forte legalismo che fu molto vicino all'atteggiamento che gli Ebrei avevano nell'ambito del culto che era puramente esteriore.

99 „Superbos longe (Deus) videt, eo illis minus propinquat, quo sibi videntur altiores. Quaerebas ergo montem? Descende ut adtingas. Sed adscendere vis? Adscende: noli montem quaerere"; Tractatus in Johannem XV,25, NBA 24/1, p. 370-371; cfr. Sermo 21,2.

100 „Quare «Pater tales quaerit qui adorent eum», non in monte, non in templo, sed «in spiritu et veritate? Spiritus est Deus. Si corpus esset Deus, oportebat eum adorari in monte, quia corporeus est mons - oportebat eum adorari in templo quia corporeum est templum. «Spiritus est Deus, et eos qui adorant eum, in spiritu et veritate oportet adorare»"; Tractatus in Johannem XV,24, NBA 24/1, p. 368-367.

101 Seguendo le orme dell'apostolo Paolo, S. Agostino sempre mette i giudei al primo posto. Cfr. L. Nieścior, Żydzi w Państwie Bożym Św. Augustyna, p. 715.

102 Tractatus in Johannem XV,26, NBA 24/1, p. 372-373.

103 Cfr. Tractatus in Johannem XV,31. 
Laccento posto sulla incorporeità di Dio è di provenienza antimanichea ${ }^{104}$. Il vero superamento della pretesa visibilità di Dio e del suo possesso in un luogo, culmina nel riconoscimento dell'assoluta trascendenza. Dio è adorato in verità soltanto se è adorato in Spirito che è imperscrutabile dono. Lo stesso valore antimanicheo hanno gli accenni alla temporaneità del santuario descritto nell'Antico Testamento sostituito dalla realtà della Chiesa ${ }^{105}$.

Il vescovo di Ippona sostiene che il vero credente con umiltà di cuore offre se stesso a Dio come tempio.

Tu pensi davvero di essere più vicino a Dio perché stai su un monte, e che più presto ti potrà esaudire, quasi tu lo invocassi da vicino? Certo, Dio abita in alto; ma „guarda le umili creature” (Sl 137,6). „Il Signore è vicino”; ma a chi? Forse a quelli che stanno in alto? No: „Il Signore è vicino a quelli che hanno il cuore contrito" $(\mathrm{Sl} 33,19)$. Cosa mirabile! Egli abita in alto, e si avvicina agli umili: „Riguarda all'umile, e da lontano conosce il superbo". Vede i superbi da lontano, e tanto meno si avvicina a loro quanto più essi si ritengono alti. E tu cercavi un monte? Discendi, se vuoi raggiungere Dio. Ma se vuoi ascendere, ascendi; solo non cercare un monte... Cerca di raccoglierti dentro di te. E se vuoi trovare un luogo alto, un luogo santo, offriti a Dio come tempio nel tuo intimo. „Santo, infatti, è il tempio di Dio, che siete voi" (1 Cor 3,17). Vuoi pregare nel tempio? Prega dentro di te; ma cerca prima di essere tempio di Dio, affinché egli possa esaudire chi prega nel suo tempio ${ }^{106}$.

Lanima attraverso la grazia può diventare il luogo di umiltà e il santuario dell'assoluta trascendenza divina ${ }^{107}$.

Soltanto con la polemica antipelagiana che gli altri riferimenti a Gv 4,23$24^{108}$, acquistano un profondo spessore teologico ${ }^{109}$. Così, ad esempio nel rapporto tra "Spiritus" e „veritas”, Agostino subordina del tutto quell'ultima allo Spirito $^{110}$. La predestinazione divina elegge alcuni e comunica a loro il dono di

104 Una lettura simile in Enarrationes in Ps. 130, 1-4.

105 Cfr. De civitate Dei XX,19.

106 Tractatus in Johannem XV,25, NBA 24/1, p. 370-371. „A lui sacrifichiamo «l'ostia» di umiltà e «di lode» (Sl 115, 17. 83) sull'altare del cuore, fiammeggiante del fuoco della carità"; De civitate Dei X, 3, 10, CCL 47, p. 275.

107 Cfr. Tractatus in Johannem C,1-4.

108 Essi sono rari e piuttosto marginali.

109 Il testo giovanneo è citato nel De Trinitate e negli ultimi scritti predestinazionistici. Il IV Vangelo si prestava non solo ai sviluppi d’argomento trinitario, ma come veniva accennato, anche nella direzione antidonatista.

110 Esso viene inteso come atto eterno della „caritas”. Bisogna ricordare che nel vangelo giovanneo „verità” è la rivelazione del Padre nella persona di Gesù. Essa si identifica con la persona del Figlio. 
Dio che è lo Spirito (cfr. At 8,20 e Gv 4,10) ${ }^{111}$. A causa dell'annuncio della verità viene rigettata la „cupiditas” umana ${ }^{112}$.

Risulta a questo punto evidente l'interpretazione dello Spirito come fonte di grazia operante che innalza l'uomo verso Dio. Si ha, quindi, ipotizzabile il motivo dello scarso entusiasmo agostiniano per Gv 4,23-24, passo che identifica "Spirito” e „verità". Solo se la verità è dono dello Spirito Santo, essa diviene conoscenza reale di Dio. In tal senso, un blocco intero di Tractatus in Johannem ${ }^{113}$ tratta del dono dello Spirito Santo: in particolare, Agostino cita Gv 16,13, ove si parla di "Spiritus veritatis”, espressione sostanzialmente coincidente con Gv 4,24. Il Paracleto con la sua potenza irresistibile offre tanti doni a chi sa accoglierli ${ }^{114}$. Lo Spirito Santo si rivela come il „maestro intimo” che dona progressivamente l'intera verità ${ }^{115}$. Qualunque pretesa di attingere la verità indipendentemente dall'atto dello Spirito, è quindi l'idolatria vera e propria. Solo l'adorazione in Spirito è quindi vera e pura, essendo generata dall'atto dell'Altro e dal suo amore senza limiti ${ }^{116}$. Dunque solo lo Spirito Santo, l'Amore assolutamente libero di Dio, può generare nell'uomo anche solo il desiderio di adorare o di pregare Dio, la verità assoluta. È certo che la libertà dell'uomo non viene annullata dalla grazia, ma accesa e vivificata per cooperare nell'amore ${ }^{117}$. Solo all'interno della Chiesa è possibile per Agostino fruire della grazia.

Nella riflessione sul Sl 130 Agostino parla del tempio di Dio in riferimento al corpo di Cristo (cfr. 1 Cor 3,17), formato dall'insieme dei fedeli.

Lessere esaudito in ordine alla vita eterna..., non è concesso se non a chi prega nel tempio di Dio. Ora nel tempio di Dio prega soltanto colui che prega nella pace della Chiesa, nell'unità del corpo di Cristo. Questo corpo di Cristo consta di molti credenti sparsi su tutta la terra, ed è per questo che chi prega nel tempio viene esaudito. Chi prega nella pace della Chiesa prega „in spirito e verità” (Gv 4,23), né la sua preghiera è fatta in quel tempio che era solamente una figura ${ }^{118}$.

111 „Procul dubio Spiritus Donum Dei est”; De Trinitate XV,17,31-19,33, PL 42, 1084; cfr. Epistula 194,4,17-5,19.

112 Cfr. Tractatus in Johannem XV,30.

113 Dal XCII al C.

114 Cfr.Tractatus in Johannem XCII,2 con riferimento a Rm 5,5.

115 Cfr. Tractatus in Johannem XCVI,6,5. Si veda anche ibidem XXXII,5; XCVII,1.

116 Sulla dipendenza dell', amor Dei” dal dono dello Spirito, cfr. Tractatus in Johannem LXXIV,1-5; 85,3;102,4-5.

117 Cfr. De dono perseverantiae 23,64.

118 Enarrationes in Ps. 130,1, NBA 28/1, p. 240-241. 
Lassemblea dei fedeli è tempio e corpo di Cristo (totus Christus). L'interpretazione in chiave cristologica permette di vedere la persona del Salvatore come unita indissolubilmente con la sua Chiesa ${ }^{119}$. Il compito dei credenti è quello di formare un edificio spirituale e radunarsi nell'unità ${ }^{120}$.

L'Ipponate enfatizza la necessità di pregare nello Spirito nel commento che segue

Nel Salmo [che stiamo trattando] risuona la voce di questo tempio... è in questo tempio che si invoca Dio in spirito e verità e lì egli esaudisce: non nel tempio materiale [del giudaismo], dove c'era soltanto un'immagine rappresentativa di ciò che sarebbe avvenuto più tardi. Lantico tempio è stato abbattuto; ma forse che per questo è rovinata anche la casa della nostra preghiera? Tutt’altro! ${ }^{121}$.

Il santuario divino non ha il carattere fisico, non è edificato dagli uomini. Esso è caduto, bisogna guardare invece la realtà spirituale della Chiesa.

Il vescovo di Ippona sottolinea che l'incontro di Dio deve andare oltre ai limiti delle umane possibilità ${ }^{122}$ perché Dio è spirito e Gli si addice un'adorazione spirituale ${ }^{123}$.

Possediamo fin dora "delle primizie dello Spirito" (cfr. Rm 8,23) e forse per altre vie ci possiamo accostare a colui che amiamo... Non è nulla di materiale. „Dio è spirito" (Gv 4,24). Perciò è scritto che "quelli che Lo adorano debbono adorarlo in spirito e verità" $(\mathrm{Gv} 4,24)$. Non in qualche luogo del corpo, perché corpo non è; non come su un monte eccelso dove tu possa pensare di accostarti a lui man mano che ci sali su. In verità eccelso è il Signore, però guarda verso il basso; mentre alle cime „volge lo sguardo da lontano” (Sl 137,6) ${ }^{124}$.

119 Cfr. De civitate Dei XVII,20.

120 „ «Il Signore ricostruisce Gerusalemme, raccoglie i dispersi del suo popolo», cioè del popolo di Gerusalemme, che poi è lo stesso che il popolo d'Israele. Cè in effetti una Gerusalemme eterna, che ha sede nei cieli e di cui son cittadini anche gli angeli"; Enarrationes in Ps. 146,4, NBA 28, p. 772-773.

121 Enarrationes in Ps. 130,3, NBA 28/1, p. 242-245.

122 Agostino parlava della trascendeza divina in Sermo 23,6, NBA 29, p. 442-443 (allusione a $G v 4,20$ ).

123 Lo fa a proposito del Sl 63.

124 Sermo 21,2, NBA 29, p. 394-395. 
Significativo in questo contesto è il richiamo di 1 Gv 4,8 (Dio è carità): amiamo ,il bene ineffabile, il bene benefico, il bene creatore di ogni bene” 125 . La riflessione prende, quindi, un'altra direzione.

Lidea di questo genere si incontra anche nel discorso 198 nella sua versione più completa ${ }^{126}$. Agostino sostiene che colui che prega bene, prega nel modo interiore (omnis homo intus orat, cum bene orat).

Per la tua preghiera [personale] tempio è il tuo cuore... Le adunanze dei fratelli incrementano dunque l'amore. Ma l'uomo che prega bene prega nel suo interno, secondo quanto rispose il Signore alla donna di Samaria: „Verrà l'ora” - le disse "ed è questa, in cui né su questo monte né a Gerusalemme adorerete il Padre" $(\mathrm{Gv} 4,21)^{127} \ldots$ Se dunque è nello spirito che bisogna adorare Dio, gli si prepari una stanza: l'ospite non mancherà di venire. Dice: „Verremo da lui, io e il Padre mio, e faremo sosta presso di lui" $(\mathrm{Gv} 14,23)^{128}$.

Non è un modo insolito di parlare del culto da rendere all'unico Dio ${ }^{129}$. Con fervore pastorale il vescovo d'Ipppona continua:

Rècati quindi in chiesa per incrementare il fervore della tua preghiera; vieni perché la devozione di unassemblea in raccoglimento ti procuri il merito per essere esaudito da Dio, non perché ci sia un qualche luogo sulla terra dove Dio risiede e ti esaudisce. Non pensare che Dio esaudisce solo da dentro le mura d'una chiesa. I martiri, [Dio] li esaudì nell'interno di un carcere! Dice: „Quale casa potreste edificarmi o quale sarà il luogo nel quale possa riposarmi? Non è stata forse la mia mano a creare tutte queste cose?" (At 7,49-50 [Is 66,1-2]). E indicò che la sua residenza è nel cuore dell'uomo fedele. Dice infatti: „Sopra chi riposerà il mio Spirito? Su chi è umile e pacifico e teme le mie parole" (Is 66,2) ${ }^{130}$.

125 „Ineffabile bonum, bonum beneficum, bonum bonorum omnium creatorem”; ibidem, NBA 29, p. 396.

126 La versione più completa venne scoperta dal Dolbeau. Cfr. F. Dolbeau (ed.), Augustin d'Hippone. Vingt-six sermons au peuple d'Afrique (CÉASA 147).

127 Segue la citazione di Gv 4, 23-24; cfr. H. Houghton, Augustine’s Text of John, p. 41 (nota 35$) ; 219$.

128 Sermo 198 [augm.], 11, NBA 35, p. 628-629.

129 „Di lui infatti siamo tempio tutti insieme e ciascuno siamo un tempio, perché Egli si degna di abitare e nella concordia di tutti e nei singoli”; De civitate Dei X, 3, 10, CCL 47, p. 275.

130 Sermo 198 [augm.], 11, NBA 35, p. 630-631. 
Agostino menziona qui „i veri adoratori”, ma non lo fa con l'enfasi delle Quaestiones ${ }^{131}$.

Le sue polemiche con alcuni eretici riguardavano le questioni connesse con la problematica del „tempio spirituale”. Un riferimento molto chiaro si può trovare nella polemica contro il vescovo ariano, Massimino.

Se lo Spirito Santo non fosse Dio, certamente non avrebbe noi come tempio. Però l'Apostolo ha scritto: „Non sapete che siete tempio di Dio e che lo Spirito di Dio abita in voi?” (1 Cor 3,16$)$ E ancora: „Non sapete che il vostro corpo è tempio dello Spirito Santo che è in voi e che avete da Dio?" (1 Cor 6,19). Se facessimo un tempio di legno e di pietra a un qualche angelo eminentissimo, non saremmo forse colpiti da anatema da parte della verità di Cristo e della Chiesa di Dio, per il fatto di riservare a una creatura quella servitù che si deve solo a Dio? ${ }^{132}$. Se, dunque, saremmo sacrileghi facendo un tempio a una creatura qualsiasi, come può non essere vero Dio colui al quale non facciamo un tempio, ma per il quale siamo tempio noi stessi? ${ }^{133}$.

Invece nel Sermo 53 Agostino insiste sul fatto che non si può immaginare Dio alla maniera fisica ${ }^{134}$. Lega questo concetto con l'idea del tempio spirituale. A questo proposito evoca Sap 1,4 e 1 Cor 3,17.

„Lanima del giusto è sede della sapienza” (Sap 1,4). Dove mai, infatti, ha Dio la propria sede, se non dove abita Lui? E dove abita, se non nel proprio tempio? Orbene, „il tempio di Dio è santo e questo tempio siete voi” (1 Cor 3,17). Vedi dunque come devi accogliere Dio. „Dio è spirito: bisogna adorare Dio in spirito e veritä” $(\mathrm{Gv} 4,24)$. Orbene, se ti aggrada, entri nel tuo cuore larca del Testamento e cada Dagone ${ }^{135}$.

La riflessione agostiniana si inserisce nel tema del contrasto fra la vita presente e quella futura ${ }^{136}$. L'Ipponate trasferisce nella sfera escatologica il riferi-

131 Cfr. Quaestiones in Heptateuchum libri septem V, 10,1, NBA 11, p. 1046-1047.

132 La Chiesa cattolica reagiva negativamente allesagerato culto degli angeli che si è mantenuto nei circoli giudeo-cristiani (cfr. Col 2,16-18) e nelle sette ereticali.

133 Collatio cum Maximino 14, PL 42, 722. Cfr. H. Houghton, Augustine's Text of John, p. 176 con note.

134 Ogni credente deve avere „un'idea spirituale di Dio, in modo da non immaginare che Dio sia corporale"; Sermo 53, 6. 7, NBA 30/1, p. 92-93. Cfr. P. Verbraken, Le Sermon 53 de saint Augustin sur les Béatitudes selon saint Mattieu, p. 24.

135 Sermo 53, 7.7, NBA 30/1, p. 92-93.

136 Agostino sosteneva che il Signore ha insegnato agli uomini a disprezzare le cose transitorie e ad amare le eterne; cfr. Sermo Mai 19, 2. 
mento al ruolo esplicito della città di Dio e al culto del vero Dio. Agostino elabora l'idea del culto in spirito e verità piuttosto nell'ambito della spreculazione teologica. Le parole del Salvatore dirette alla Samaritana sono in armonia con la realtà attuale del culto cristiano, spesse volte non solo menzionato dal Dottore latino, ma anche da lui descritto ${ }^{137}$.

\section{Conclusioni}

La letteratura cristiana dimostra che Gv 4,21-24 permetteva di sviluppare diverse problematiche, ma non di rado è stata fraintesa con l'attribuzione di significati lontani dal suo valore reale. „Adorare in Spirito e verità" è espressione che aveva molte interpretazioni nella storia dell'esegesi. I Padri vanno ben oltre alle interpretazioni scontate secondo cui il culto da rendere a Dio non va più legato a un luogo esteriore o a un tempio, ma deve essere spirituale, interiore. Nei loro scritti si sono intrecciate non solo le questioni di interpretazione biblica. Si sono visti gli autori di provenienza antiochena nei diversi contesti storico-dottrinali (Giovanni Crisostomo, Teodoro di Mopsuestia e Teodoreto di Cirro). Invece sull'altra sponda si è trovato Cirillo Alessandrino. Ai Padri orientali viene accostato Girolamo, ma soprattutto Agostino di Ippona. I commentatori antichi interpretavano il culto spirituale come „l'intimo tempio del proprio cuore", ma vedevano il valore nella realtà concreta di Gerusalemme. Le affermazioni pronunciate dal Signore nel dialogo con la Samaritana sono la conferma che alla capitale di Giudea non va più conferito uno „status” speciale. È vero che il culto autentico parte dal cuore, tuttavia è anche vero che unadorazione non soltanto individuale ma comunitaria si esprime in un luogo e con riti esteriori. L'impiego dei termini presenti in Gv 4,21-24 evidenzia che questo testo fu capace di catalizzare questioni di decisiva portata. Si trattava non solo dei problemi esegetici o rituali, ma anche delle questioni filosofiche, storiche e spirituali. I commentatori di questo antichi hanno indicato il culto „in Spirito e verità” come un tratto caratteristico del cristianesimo. Tuttavia, i primi scrittori cristiani videro anche le implicazioni specifiche di questa verità e del suo impatto sulla vita cristiana concreta.

137 Cfr. P.F. Beatrice, Culto cristiano, in: A. Fitzgerald, Agostino. Dizionario enciclopedico, p. 514-523. 


\section{Bibliografia}

\section{Fonti}

Augustinus Hipponensis, Collatio cum Maximino, ed. J.P. Migne, Patrologiae cursus completus: Series Latina [= PL] 42, 709-742.

Augustinus Hipponensis, Confessiones, Nuova Biblioteca Agostiniana [= NBA] 1, ed. M. Pellegrino, C. Carena, Roma 1975.

Augustinus Hipponensis, De civitate Dei, ed. A. Trapé, D. Gentili, NBA 9 B, Roma 1988. Augustinus Hipponensis, De dono perseverantiae. PL 45, 993-1034.

Augustinus Hipponensis, De Trinitate, ed. W.J. Mountain, Corpus Christianorum, Series Latina [= CCL] 50-50A, Turnhout 1968.

Augustinus Hipponensis, Enarrationes in Psalmos, NBA 27-28, ed. V. Tarulli, T. Mariucci, F. Monteverde, Roma 1976-1977.

Augustinus Hipponensis, In Evangelium Joannis tractatus, ed. O. Campagna, E. Gandolfo, V. Tarulli, F. Monteverde, NBA 24/1-2, Roma 20043.

Augustinus Hipponensis, In I epistola Joannis, ed. P. Agaësse, Sources Chrétiennes [= SCh] 75, Paris $2011^{3}$.

Augustinus Hipponensis, Quaestiones in Heptateuchum libri septem, ed. L. Carrozzi, A. Pollastri, NBA 11/1, Roma 1997.

Augustinus Hipponensis, Sermones, NBA 29-34, ed. P. Bellini, L. Carrozzi, F. Cruciani, Roma 1983-1984; 1989; ed. F. Dolbeau, Augustin d'Hippone. Vingt-six sermons au peuple d'Afrique (CÉASA 147), Paris 1996.

Cyrillus Alexandrinus, Commentarii in Joannem, ed. J.-P. Migne, Patrologiae cursus completus: Series Graeca [= PG] 73, 9-756; 74, 9-104.

Cyrillus Alexandrinus, Commentarius in XII prophetas minores, PG 71, 9-1061; 72, 9-364.

Cyrillus Alexandrinus, Contra Julianum, ed. P. Burguière, P. Évieux, SCh 322, Paris 1985.

Cyrillus Alexandrinus, De adoratione et cultu in spiritu et veritate, PG 68, 133-1125.

Cyrillus Alexandrinus, In Isaiam, PG 70, 9-1450.

Hieronymus Stridonensis, Commentarii in Prophetas, ed. M. Adriaen, CCL 76; 76 A, Turnhout 1969-1970.

Hieronymus Stridonensis, Commentarius in Esaiam, ed. M. Adriaen, G. Morin, CCL 73; 73A, Turnhout 1963.

Hieronymus Stridonensis, Epistulae, ed. J. Labourt, Saint Jérôme. Lettres/I-VIII, vol. 1-8 (Collection des Universités de France), Paris 1950-1958.

Joannes Chrysostomus, Expositiones in Psalmos, PG 55, 39-498.

Joannes Chrysostomus, In Joannem homiliae, trad. ing. Ph. Schaff, A Select Library of the Nicene and Post-Nicene Fathers of the Christian Church (= NPNF) ser. I, vol. 14, Peabody 1994 (reprint),

Lactantius, Epitome Divinarum Institutionum ed. P. Monat, SCh 335, Paris 1987. 
Sermones Escorial, ed. A. Hamman, Patrologia Latina Supplementum [= PLS] 4, 669740.

Tertullianus, De oratione, ed. G.F. Diercks, CCL 1, Turnhout 1954.

Theodoretus Cyrensis, Epistulae, ed. Y. Azéma, vol. 1-3, SCh 40; 98 111, Paris 1955; $1964 ; 1965$.

Theodoretus Cyrensis, Eranistes seu Polymorphus, PG 85, 27-336, trad. ingl. G.H. Ettinger, Theodoret of Cyrus. Eranistes, Oxford 1975.

Theodoretus Cyrensis, Explanatio in Ezechielem, PG 81, 807 A - 1256 B.

Theodoretus Cyrensis, Historia religiosa, ed. P. Canivet et al., vol. 1-2, SCh 234; 257, Paris $1977 ; 1979 .$.

Theodorus Mopsuestenus, Commentaria in Isaiam, ed. J.-N. Guinot, vol. 1-3, SCh 295; 276; 315, Paris 1980; 1982; 1984.

Theodorus Mopsuestenus, Commentaria in prophetas minores, PG 66, 123-632.

Theodorus Mopsuestenus, Commentarius in evangelium Johannis Apostoli, ed. J.M. Vosté, CSCO 115-116, Louvain 1940.

\section{Studi}

Ashbyl G.W., Theodoret of Cyrrhus as Exegete of the Old Testament, Grahamstown (South Africa) 1972.

Beatrice P.F., "Culto cristiano," in: A. Fitzgerald, Agostino. Dizionario enciclopedico, trad. it. L. Alici, A. Pieretti, Roma 2007, p. 514-523.

Bori P.C. (a cura di), In spirito e verità. Lettura di Giovanni 4,23-24 (L'Epifania della Parola 6), Bologna 1996.

Braun F.-M., Jean le Théologien et son Évangile dans l'Église ancienne, Paris 1959.

Chadwick H., The Church in Ancient Society: From Galilee to Gregory the Great (Oxford History of the Christian Church), Oxford 2001.

Cristiani M., Il Prologo di Giovanni da Agostino a Tommaso d’Aquino, Annali di storia dell'esegesi 11/1 (1994), p. 52-53.

De Margerie B., Introduzione all storia dell'esegesi, vol. 3: S. Agostino, Roma 1986.

Devreesse R., Essai sur Théodore de Mopsueste (Studi e Testi 141), Città del Vaticano 1948.

Dubois J. D., „Jean dans l'Eglise ancienne," Le Monde de la Bible 53 (1988), p. 38-40.

Fatica L., I Commentari a Giovanni di Teodoro di Mopsuestia e di Cirillo di Alessandria, Roma 1988.

Grabau J. L., John 4:23-24 in North African Preaching, Scrinium 13 (2017), p. 138-140. Gribomont J., Lorigenisme de Saint Basile, in: L'homme devant Dieu. Mélanges H. de Lubac, Paris 1963, p. 281-294.

Guinot J.-N., L'éveque exégète: Théodoret de Cyr (BTT 1), Paris 1984.

Guinot J.-N., L'exégèse de Théodoret de Cyr (Théologie Historique 100), Paris 1995.

Houghton H., Augustine's Text of John. Patristic Citations and Latin Gospel Manuscripts, Oxford 2008. 
Hunt E.D., Holy Land Pilgrimage in the Later Roman Empire AD 312-460, Oxford 1982.

Lettieri G., "In spirito e/o verità da Origene a Tommaso d'Aquino," Annali di storia dell'esegesi 12/1 (1995), p. 49-83.

Lettieri G., "In spirito e/o verità. Da Origene a Tommaso d'Aquino," in: P.C. Bori (a cura di), In spirito e verità. Lettura di Giovanni 4,23-24 (L'Epifania della Parola 6), Bologna 1996, p. 69-70.

Paczkowski M.C., "Gerusalemme - «ombelico del mondo» nella tradizione cristiana antica”, Liber Annuus SBF 55 (2005), p. 165-202.

Paczkowski M.C., "Gerusalemme in Origene e San Girolamo," in: G. Bissoli (ed.), Gerusalemme. Realtà sogni speranze, Jerusalem 1996, p. 115-123

Perrone L., “"Sacramentum Iudeae» [Gerolamo, Ep. 46]: Gerusalemme e la Terra Santa nel pensiero cristiano dei primi secoli. Continuità e trasformazioni, in: Melloni A., Menozzi D., Ruggieri G., Toschi M. (ed.), Cristianesimo nella storia. Saggi in onore di G. Alberigo, Bologna 1996, p. 445-478.

Perrone L., "«The Mystery of Judaea» (Jerome, Ep. 46): The Holy City of Jerusalem between History and Symbol in Early Christian Thought," in: L.I. Levine (ed.), Jerusalem. Its Sanctity and Centrality to Judaism, Christianity and Islam, New York 1999, p. 221-239.

Pesce M., Destro A., "Lo Spirito e il mondo «vuoto». Prospettive esegetiche e antropologiche su Gv 4,21-24," Annali di storia dell'esegesi 12/1 (1995), p. 9-32.

Poorthuis M., Safrai C. (ed.), The centrality of Jerusalem. Historical perspectives, Kampen 1996.

Quasten J., Patrologia, vol. 3, Casale Monferrato 1983.

Rigato M.L., "Gv 4: La mente cultuale dell'Evangelista. Gesù si rivela alla donna Samaritana," in: L. Padovese (a cura di), Atti del V Simposio di Efeso su S. Giovanni Apostolo, Roma 1995, p. 27-84.

Russell N., Cyril of Alexandria (The Early Church Fathers), London-New York 2000.

Sartoni C., Gesù dono di Dio, sorgente di acqua viva. Meditazioni esegetiche a Gv 4,142, Imola 2007.

Simonetti M., Lettera e/o allegoria. Un contributo alla storia dell'esegesi patristica (Studia Ephemeridis „Augustinianum” 23), Roma 1985.

Verbraken P., "Le Sermon 53 de saint Augustin sur les Béatitudes selon saint Mattieu," Revue Bénédictine 104 (1994), p. 19-33.

Walker P.W.L., Holy City, Holy Places? Christian Attitudes to Jerusalem and the Holy Land in the Fourth Century (Oxford Early Christian Studies 2), Oxford 1990.

Wiles M., The Spiritual Gospel. The Interpretation of the Fourth Gospel in the early Church, Cambridge 1960.

Wilken R.L., The Land Called Holy. Palestine in Christian History and Thought, New Haven-London 1992. 